\title{
A radiobiological comparison of hypo-fractionation versus conventional fractionation for breast cancer 3D-conformal radiation therapy
}

\author{
Arezoo Kazemzadeh ${ }^{1}$, Iraj Abedi ${ }^{2}$, Alireza Amouheidari ${ }^{3}$, Atefeh Shirvany ${ }^{4}$ \\ ${ }^{1}$ Kashan University of Medical Sciences, Kashan, Iran \\ ${ }^{2}$ Medical Physics Department, Isfahan University of Medical Sciences, Isfahan, Iran \\ ${ }^{3}$ Isfahan Milad Hospital, Isfahan, Iran \\ ${ }^{4}$ Isfahan University of Medical Sciences, Isfahan, Iran
}

\begin{abstract}
Background: The present research was aimed to compare the toxicity and effectiveness of conventional fractionated radiotherapy versus hypo-fractionated radiotherapy in breast cancer utilizing a radiobiological model.

Materials and methods: Thirty-five left-sided breast cancer patients without involvement of the supraclavicular and axillary lymph nodes (with the nodal stage of N0) that had been treated with conventional or hypo-fractionated were incorporated in this study. A radiobiological model was performed to foretell normal tissue complication probability (NTCP) and tumor control probability (TCP).

Results: The data represented that TCP values for conventional and hypo-fractionated regimens were $99.16 \pm 0.09$ and 95.96 \pm 0.48 , respectively $(p=0.00)$. Moreover, the NTCP values of the lung for conventional and hypo-fractionated treatment were 0.024 versus $0.13(p=0.035)$, respectively. Also, NTCP values of the heart were equal to zero for both regimens.

Conclusion: In summary, hypo-fractionated regimens had comparable efficacy to conventional fraction radiation therapy in the case of dosimetry parameters for patients who had left breast cancer. But, utilizing the radiobiological model, conventional fractionated regimens presented better results compared to hypo-fractionated regimens.
\end{abstract}

Key words: radiobiological model; hypo-fractionation; conventional fractionation; breast cancer; 3D-conformal radiation therapy Rep Pract Oncol Radiother 2021;26(1):86-92

\section{Introduction}

Radiation therapy is an important part of curative treatment for majority of breast cancer patients. Traditionally, in conventionally fractionated (CF) breast cancer radiation therapy a dose of $2 \mathrm{~Gy}$ in 25 daily fractions is prescribed (CF). Such a long treatment schedule has major implications on both patient's compliance and department workload, especially in a country like Iran where workforce and resources are always a constraint. Lately, a task force principle has been published by the Radiation Oncology American Society, suggesting hypofractionated radiotherapy (HR) for all women at any age, whether they had had chemotherapy or not, over the past decades [1]. Studies from the United Kingdom and Canada supported their evidence-based suggestions. There has been a growing trend toward HF which involves delivering a higher dosage per fraction in a smaller number of frac- 
tions (2.8 Gy daily in 15 fractions) for a biologically equivalent dose, while maintaining the same toxicity and loco-regional control rates $[2,3]$. The previous studies, mostly used follow-up trials to investigate these two regimens $[2,4-6]$. Recently, radiobiological models have been utilized only to predict the extra risk of second cancer after radiation therapy [7-9].

Given that radiobiological models are able to predict the effects of fraction size on radiation therapy of breast cancer [9], this research is aimed to examine the radiobiological effect of hypo-fractionated radiation therapy in comparison to conventional fraction in patients with left breast cancer.

\section{Materials and methods}

\section{Patients}

This retrospective study was performed at the department of radiation oncology, Milad Hospital, Isfahan, Iran. Thirty-five left-sided breast cancer patients without involvement of the supraclavicular and axillary lymph nodes (with the nodal stage of N0) that had been treated with conventional or hypo-fractionated were incorporated in this study.

\section{Contouring and planning objectives}

The organs at risk (OARs) contouring was performed according to the International Commission on Radiation Units and Measurements (ICRU) reports 50, 62 and 83 [10]. The clinical target volume (CTV) for the lymph node (LN), chest wall, and breast were contoured with reference to the Radiation Therapy Oncology Group (RTOG) contouring atlas [11]. The planning target volumes (PTVs) were attained by 5 millimeter extension in all directions from the clinical target volumes and were also limited to have a trim of 3 millimeters from the surface in the case of breast clinical target volume.

The prescribed dose for conventional and hypofractionated RT were 50 Gy at 2 Gy and 4.05 at 2 Gy per fraction to the PTVs, respectively. The main goal in RT planning was to deliver 95 percent of the dose prescribed in 95 percent of the planning target volumes. For planning confirmation in conventional RT, PTV $\mathrm{D}_{95 \%} \geq 47.8 \mathrm{~Gy}$, the highest dose $\left(D_{2 \%}\right) \leq 55.4$ Gy to the planning target volume, and in hypo-fractionated RT, PTV $\mathrm{D}_{95 \%} \geq 38.6 \mathrm{~Gy}$, the highest dose $\left(\mathrm{D}_{2 \%}\right) \leq 44.8$ Gy to the planning target volume. The lowest dose to 95 percent of PTVs that is not lower than 95 percent of their respective doses prescribed were needed.

The dose of irradiation to the OARs was limited as below: the planning aims were an average lung dose of 15 Gy with $\mathrm{V}_{20 \mathrm{~Gy}}$ less than 30 percent (no more than 30 percent of the organs at risk volume receiving $20 \mathrm{~Gy}$ ) for the lungs and $\mathrm{V}_{25 \mathrm{~Gy}}$ less than 20 percentages for the heart.

\section{Planning techniques}

The RT plans were generated using PROWESS PANTER version 5.5, and all plans were made for treatment on an ARTISTE radiation therapy system (SIEMENS). Two tangential beams (6 MV photons) with a $15^{\circ}$ wedge angle were used for 3D-CRT. The weights of these two opposed tangential beams were arranged to allocate the hot spots and deliver a homogeneous dose to the purpose volume. The Beam shaping was accomplished with multi-leaf collimators (MLC) and gantry angles of beams adjusted to protect OARs.

\section{Plan evaluation}

Dose-volume histogram (DVH) analysis is used to evaluate the plan. The average doses and values of $\mathrm{V}_{110 \%}$ and $\mathrm{V}_{105 \%}$ (the PTV percent receiving at least 110 and 105 percent of the prescribed dose, respectively), $D_{\text {mean }}, D_{\max }$ (average dose), $D_{2 \%}$ and $D_{98 \%}$ (minimum dose to 2 and $98 \%$ of the planning target volume) were reported for planning target volume.

The conformity index (CI) and the dose homogeneity index (HI) were computed utilizing the below definitions, and the closer the values of $\mathrm{HI}$ and $\mathrm{CI}$ are to 1, the better the conformal coverage:

$$
C l=B V 95 \% / P T V \text { volume }
$$

$\left(\mathrm{BV}_{95 \%}=\right.$ body volume of the isodose of 95 percent of the prescribed dose)

$$
H I=D 5 \% / D 95 \%
$$

$\left(D_{5 \%}=\right.$ the lowest dose to $5 \%$ of the planning target volume, $\mathrm{D}_{95 \%}=$ the lowest dose to $95 \%$ of the planning target volume)

To assess the dose irradiated to OARs, the analysis contained the average dose and $\mathrm{V}_{\mathrm{XGY}}$ (organs at risk volume receiving $X \mathrm{~Gy}$ ), depending on the organ. For the lung, radiation pneumonitis (RP) incidence was less than 20 percent when the average 
Table 1. The radiobiological parameters used for left breast irradiation

\begin{tabular}{|l|c|c|c|c|}
\hline Organ & $\mathrm{a}$ & $\mathrm{Y}_{50}$ & $\mathrm{TD}_{50}$ (Gy) & $\mathrm{TCP}_{50}(\mathrm{~Gy})$ \\
\hline Heart & 3 & 3 & 48 & - \\
\hline Lung & 1 & 2 & 24.5 & - \\
\hline PTV & -7.2 & 2 & - & 28 \\
\hline
\end{tabular}

TD - tumor dose; TCP — tumor control probability; PTV — planning target volumes

lung dose was less than about $20 \mathrm{~Gy}$. According to threshold of $\mathrm{V}_{\text {dose }}$ models, $\mathrm{RP}$ risk was less than $20 \%$ for V $5<60 \%$ with conventional fractionation or $\mathrm{V} 20<30-35 \%$. For the heart, $\mathrm{D}_{\text {mean }}, \mathrm{V}_{25 \mathrm{G} y}$ and $\mathrm{V}_{30 \mathrm{~Gy}}$ were compared.

\section{Radiobiological assessing}

The equivalent uniform dose (EUD) mathematical model was derived based on a mechanistic formulation utilizing a linear quadratic cell survival model [12]. Equivalent dose was calculated by equation 3.

$$
E U D=\left(\sum V_{i} D_{i}^{a}\right)^{\frac{1}{a}}
$$

Where vi is unitless representing the i'th partial volume receiving dose $\mathrm{D}_{\mathrm{i}}$ in $\mathrm{Gy}$ and $\mathrm{a}$ is a unitless model parameter which is specific to the tumor of interest or normal structure.

Following equations are used to evaluate the tumor control probability (TCP) and normal tissue complication probability (NTCP):

$$
\begin{gathered}
N T C P=\frac{1}{1+\left(\frac{T D_{50}}{E U D}\right)^{4 \gamma}} \\
T C P=\frac{1}{1+\left(\frac{T C D_{50}}{E U D}\right)^{4 \gamma}}
\end{gathered}
$$

Where $\gamma_{50}$ is a unitless model parameter which is specific to tumor of interest or the normal structure and explains the slope of the curve of dose response and the $\mathrm{TD}_{50}$ is the tolerance dose for complication rate of $50 \%$ at a particular time interval when the entire interest organ is irradiated homogeneously. The $\mathrm{TCD}_{50}$ is the tumor dose to manage 50 percent of the tumors when the tumor is irradiated homogeneously.

For biological evaluation of our plans, we used Bioplan (ver.1.3.3) software developed by SanchezNieto and Nahum [13]. This software was provided freely by developers on a personal request. Differ- ential dose volume histograms (dDVHs) were calculated for the PTV, Heart and Lung. DVHs were sent out as ASCII files for Bioplan with a dose bin width of $0.25 \mathrm{~Gy}$, corresponding to a total of about 333 bins; volumes were given in $\mathrm{cm}^{3}$.

Table 1 lists the radiobiological parameters used for left breast irradiation in both regimens, using the EUD based model.

\section{Statistical analyses}

SPSS program statistical package, version 22 (SPSS, Illinois, Chicago, USA) was used to analyze data. The paired student's t-test was utilized for assessing the difference between the two regimens. The significant level was considered at 0.05 . Also, data was analyzed by calculating means, range, median, and standard deviations. The data were presented as the mean $\pm \mathrm{SD}$.

\section{Results}

\section{Target volume}

The results of target doses include the mean dose and HI, TCP, and EUD for the conventional and hypo-fractionated regimens are listed in Table 2. As can be seen, the conventional RT had higher mean dose than hypo-fractionated RT (51.404 \pm 0.71 and $41.641 \pm 0.57$, respectively, $\mathrm{p}<0.001)$. Meanwhile, in terms of $\mathrm{HI}$ both regimens show almost similar outcomes. The standard deviations and average of TCP for conventional and hypo-fractionated left breast treatment were $99.16 \pm 0.09$ and $95.96 \pm 0.48$, respectively, indicating a statistically significant level. In addition, the EUD for conventional RT $(50.91 \pm 0.7)$ was significantly higher compared to hypo-fractionated RT $(41.24 \pm 0.6)$ $(\mathrm{p}$-value $=0.000)$.

\section{Organs at risk (OAR)}

The results of EUD and NTCP for normal tissues are shown in Table 3 . In general, there was no meaningful difference between the hypo-fraction and the 
Table 2. The results of tumor control probability (TCP), the equivalent uniform dose (EUD), mean dose, the conformity index (CI) and the dose homogeneity index (HI) for target volume

\begin{tabular}{|l|c|c|c|}
\hline$p$-value & Hypo-fractionated & $50.91 \pm 0.7$ & Plan \\
\hline 0.000 & $41.24 \pm 0.6$ & $99.16 \pm 0.09$ & TCP (Gy) \\
\hline 0.000 & $95.96 \pm 0.48$ & $51.404 \pm 0.71$ & Mean dose (Gy) \\
\hline 0.000 & $41.641 \pm 0.57$ & $0.1821 \pm 0.005$ & $\mathrm{HI}$ \\
\hline 0.996 & $0.1822 \pm 0.005$ & $0.89 \pm 0.03$ & $\mathrm{Cl}$ \\
\hline 0.86 & $0.89 \pm 0.03$ & & \\
\hline
\end{tabular}

Table 3. The normal tissue complication probability (NTCP) and equivalent uniform dose (EUD) values of conventional and hypo-fractionated radiotherapy (RT) for organs at risk (OARs)

\begin{tabular}{|c|c|c|c|c|c|c|}
\hline \multirow{2}{*}{ OAR } & \multicolumn{2}{|c|}{ NTCP } & & \multicolumn{2}{|c|}{ EUD } & \multirow[b]{2}{*}{ p-value } \\
\hline & CF & HF & p-value & CF & HF & \\
\hline Heart & 0.00 & 0.000 & 0.061 & $12.93 \pm 6.34$ & $10.47 \pm 5.13$ & 0.079 \\
\hline Left lung & $0.024 \pm 0.06$ & $0.13 \pm 0.33$ & 0.035 & $7.97 \pm 3.23$ & $6.45 \pm 2.62$ & 0.035 \\
\hline
\end{tabular}

CF - conventionally fractionated; $\mathrm{HF}$ - hypo-fractionated

conventional regimens for EUD in the heart (12.93 \pm 6.34 vs. $10.47 \pm 5.13$, respectively ( $\mathrm{p}>0.05)$. Also, the calculated NTCP was zero for both treatments. According to the results, the NTCP of the lung for conventional and hypo-fractionated RT were 0.024 \pm 0.06 and $0.13 \pm 0.33$, respectively. In addition, the average EUD values were higher for conventional compared to hypo-fractionated regimens (7.97 \pm 3.23 vs. $6.45 \pm 2.62$, respectively).

Table 4 summarizes some important dosimetric parameters of the heart and lung for the two RT plans. Lung mean dose was higher for CF than for HF $[7.76 \pm 0.5 v s .6 .28 \pm 0.4(\mathrm{p}<0.05)]$. In addition, for the heart, there was not much of a difference in terms of mean dose between the two regimens $(\mathrm{p}=0.299)$. Specifically, V20 of the lung showed no significant difference in CF and HF ( $\mathrm{p}>0.05)$. Moreover, we found that the heart V30 of CF and HF regimens were 23.4 (4.3) and 18.54(3.6), respectively ( $\mathrm{p}>0.05)$.

Figure 1 shows the distribution of dose and the dose-volume histogram (DVH) for one of the pa- tients who underwent hypo-fractionated and conventional RT.

\section{Discussion}

The standard radiation treatment schedule after breast conservative surgery is 50 Gy in 25 daily fractions of 2 Gy over 5 weeks. However, a shorter treatment scheme (like 40.5 Gy in 15 fractions managed within approximately 3 weeks) is both safe and equally beneficial in these cases. Outcomes of randomized trials comparing routine and moderately hypo-fractionated (13-16 fractions) radiotherapy schemes containing a total of 7,000 patients have not indicated obvious differences in efficacy of treatment, incidence of complications of late post-radiotherapy or cosmetic influences during a 5-10-year follow-up [14-16]. The Ontario [17], due to its long term follow-up, provided an especially strong evidence of non-inferiority of this method in comparison with conventionally fractionated radiotherapy. This result is also supported

Table 4. Dosimetric parameters of the lung and heart for conventional and hypo-fractionated schedules

\begin{tabular}{|l|c|c|c|c|c|c|}
\multirow{2}{*}{ Organ } & \multicolumn{7}{c}{ Lung } \\
\cline { 2 - 7 } & Conventional & \multicolumn{2}{c}{ Hypo-fraction } & p-value & Conventional & \multicolumn{2}{c}{ Hypofraction } & p-value \\
\hline Mean dose & $7.76 \pm 0.5$ & $6.28 \pm 0.4$ & 0.042 & $3.24 \pm 0.4$ & $2.62 \pm 0.3$ & 0.299 \\
\hline V20 & $20.99 \pm 3.4$ & $14.55 \pm 6.4$ & 0.283 & - & - & - \\
\hline V30 & - & - & - & $23.4 \pm 4.3$ & $18.54 \pm 3.6$ & 0.359 \\
\hline
\end{tabular}



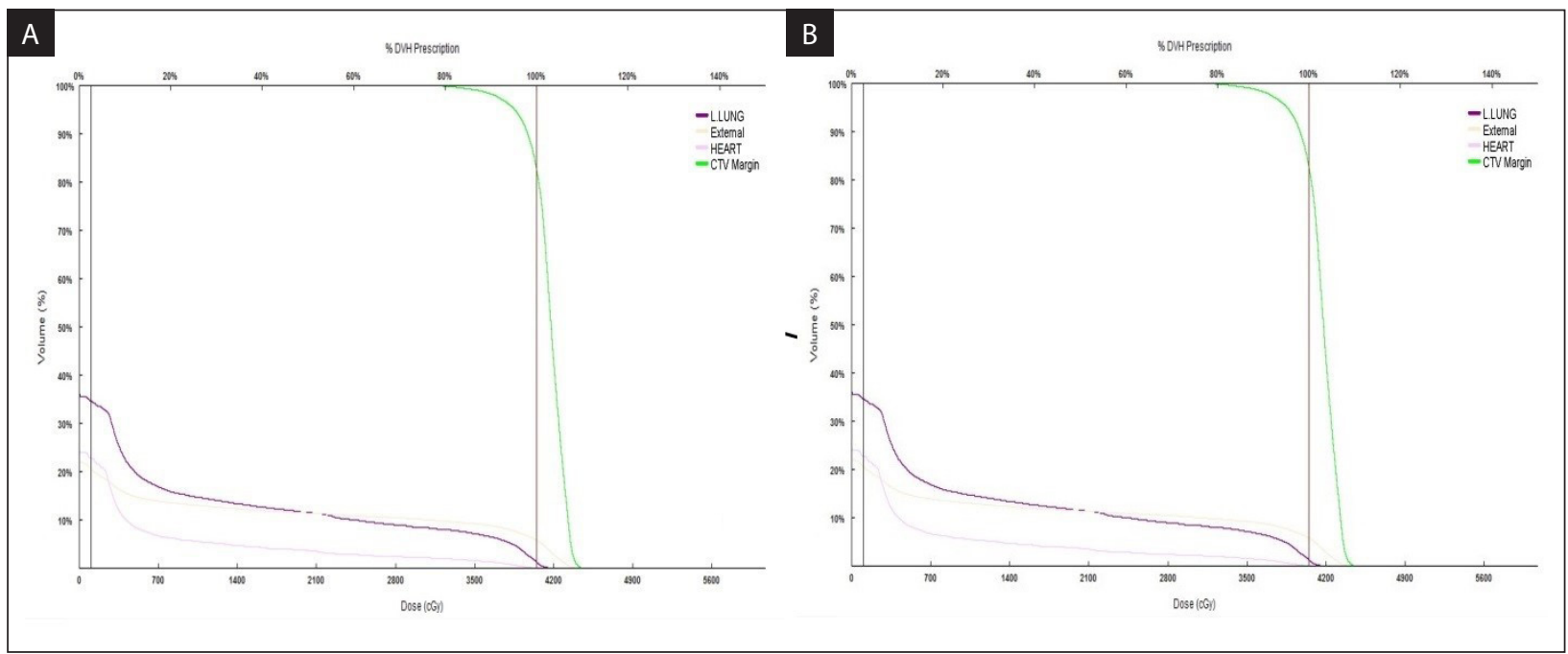

Figure 1. Dose-volume histograms for: A. Hypo-fraction and, B. Conventional fraction in left breast cancer radiotherapy

by findings of the three huge completed UK research works. The late consequences of radiotherapy containing just five large fractions for five weeks have not been established. However, there are some suspicions about whether hypo-fractionated plans can be safely performed as a regular practice in all breast cancer patients.

According to Hall et al. [18], "laboratory data demonstrated that, although early reactions match an appropriate adjustment of the total dose, large and fewer dose fractions lead to more intense late reactions. Hall also states that "the treatment protocol with several large fractions lead to more intense late influences, if the total dose is titrated to create equal early influences and a fractionated scheme is altered in clinical practice from multiple small doses to a several large fractions". Thus, according to Hall, fraction size "is the main factor in assigning late influences, the overall duration of treatment has little effect".

Recently, some clinical trials have shown that hypo-fractionated RT is equivalent to conventionally fractionated RT in terms of survival and local control outcomes and acute toxicity results $[8,19,20]$. In addition, hypo-fractionated radiation therapy suggests benefits in terms of treatment delivery and patient time. In present study, using the Niemierko's model, we aimed to assess the potential benefits that could arise from hypo-fractionated and conventional fraction for breast cancer patients. We performed a radiobiological evaluation to calculate EUD, NTCP, and TCP. Table 2 shows the results of the TCP and EUD values that were statistically higher for conventional compared to hypo-fractionated RT ( $p$-value $<0.005$ ), in which they were in contrast with reports by Gloi et. al. [21].

Data from previous observations indicate that radiotherapy related cardiovascular toxicity reveals only ten years after treatment. Therefore, there are concerns that the 5-10 years of follow-up may be too short to fully assess the hypo-fractionated radiotherapy safety for primary breast cancer. In our study, for the heart, similar NTCP equal to zero was estimated for both regimens. Paired t-test showed a p-value of 0.061 for the difference between the two techniques. Also, the hypo-fractionated scheme for lung doses has a lower V20 than conventional treatment in our study, there was no statistical difference between the two regimens in terms of V20 $(\mathrm{p}=$ 0.283 ). We found that despite the lack of significant V20 differences between the two fractionation schemes, a meaningful difference was observed in the NTCP of the lung $(0.024 \pm 0.06$ for CF vs. 0.13 \pm 0.33 for $\mathrm{HF}, \mathrm{p}$-value $=0.035$ ). The fractioned regimens in radiotherapy are based on higher repair of sub-lethal damages in normal tissue in comparison to tumor cells between the fractions [22]. In the case of biological evaluation based on the EUD model, we found that treatment with HF regimens showed acceptable side effects for OARs.

Based on our results, the dosimetric parameters including $\mathrm{HI}$ and $\mathrm{CI}$ in patients who had left breast cancer indicated no meaningful difference between the two groups, which was in agreement with Gloi 
et. al. [21] findings. Several studies have analyzed dose-volume parameters to find any predictive factor of heart and lung toxicity [23-26]. In the current study, there was no statistical difference between the treatment regimens for the heart $(p>0.05)$, but in the case of the lung, the difference was meaningful $(\mathrm{p}=0.042)$. This result was in accordance with a recent research by Mazonaski et al. [23], in which the lung dose in standard fractionated plans had higher values compared to those hypo-fractionated.

Generally, the use of the TCP/NTCP model allows predicting the scheme of prescription of dose for patients, But this research is restricted by the few number of breast cancer patients participating in this work and lack of follow-up effort. Moreover, dosimetric parameters derived from DVHs, which are usually based on a computed tomography (CT) scan with a single planning that does not account for anatomic changes during radiotherapy.

\section{Conclusion}

In summary, hypo-fractionated regimens had comparable efficacy to conventional fraction radiation therapy in terms of dosimetry parameters for patients who had left breast cancer. But utilizing the radiobiological model, conventional fractionated presented better results compared to hypofractionated regimens.

\section{Conflict of interest}

The authors of this article have no conflicts of interest to report.

\section{Funding}

This research did not receive any specific grant from funding agencies in the public, commercial, or not-for-profit sectors.

\section{References}

1. Bolukbasi Y, Selek U. Modern Radiotherapy Era in Breast Cancer. In: Pham PV, Selek U. ed. Breast Cancer: From Biology to Medicine. IntechOpen, London 2017: 269.

2. James M, Swadi S, Yi Ma, et al. Ischaemic heart disease following conventional and hypofractionated radiation treatment in a contemporary breast cancer series. J Med Imaging Radiat Oncol. 2018;62(3):425-431, doi: 10.1111/17549485.12712, indexed in Pubmed: 29436171.

3. Kawaguchi $\mathrm{H}$, Tsujino K, Miki M, et al. Patient preference study comparing hypofractionated versus conventionally fractionated whole-breast irradiation after breast- conserving surgery. Jpn J Clin Oncol. 2019; 49(6):545-553, doi: 10.1093/jjco/hyz003, indexed in Pubmed: 30796835.

4. Fragkandrea I, Kouloulias V, Mavridis $\mathrm{P}$, et al. Radiation induced pneumonitis following whole breast radiotherapy treatment in early breast cancer patients treated with breast conserving surgery: a single institution study. Hippokratia. 2013; 17(3): 233-238, indexed in Pubmed: 24470733.

5. Khan M, Siddiqui SA, Gupta MK, et al. Normal Tissue Complications following Hypofractionated Chest Wall Radiotherapy in Breast Cancer Patients and Their Correlation with Patient, Tumor, and Treatment Characteristics. Indian J Med Paediatr Oncol. 2017; 38(2): 121-127, doi: 10.4103/ ijmpo.ijmpo_80_16, indexed in Pubmed: 28900318.

6. Chan EK, Woods R, Virani S, et al. Long-term mortality from cardiac causes after adjuvant hypofractionated vs. conventional radiotherapy for localized left-sided breast cancer. Radiother Oncol. 2015; 114(1): 73-78, doi: 10.1016/j. radonc.2014.08.021, indexed in Pubmed: 25227961.

7. Hostova B, Matula P, Dubinsky P. Prediction of toxicities of prostate cancer radiotherapy. Neoplasma. 2016; 63(1): 163-168, doi: 10.4149/neo_2016_020, indexed in Pubmed: 26639247.

8. Kouloulias V, Mosa E, Zygogianni A, et al. A Retrospective Analysis of Toxicity and Efficacy for 2 Hypofractionated Irradiation Schedules Versus a Conventional One for Post-Mastectomy Adjuvant Radiotherapy in Breast Cancer. Breast Care (Basel). 2016; 11(5): 328-332, doi: 10.1159/000449433, indexed in Pubmed: 27920625.

9. Sukhikh ES, Sukhikh LG, Taletsky AV, et al. Influence of SBRT fractionation on TCP and NTCP estimations for prostate cancer. Phys Med. 2019; 62: 41-46, doi: 10.1016/j. ejmp.2019.04.017, indexed in Pubmed: 31153397.

10. Sun $Y, Y u X L$, Luo W, et al. Recommendation for a contouring method and atlas of organs at risk in nasopharyngeal carcinoma patients receiving intensitymodulated radiotherapy. Radiother Oncol. 2014; 110(3): 390-397, doi: 10.1016/j.radonc.2013.10.035, indexed in Pubmed: 24721546.

11. White J, Tai A, Arthur S, et al. Breast Cancer Atlas for Radiation Therapy Planning: Consensus Definitions. https://www.nrgoncology.org/Portals/0/Scientific\%20Program/CIRO/Atlases/BreastCancerAtlas_corr. pdf?ver=2018-04-18-144201-270 (October, 2019).

12. Gay HA, Niemierko A. A free program for calculating EUD-based NTCP and TCP in external beam radiotherapy. Phys Med. 2007; 23(3-4): 115-125, doi: 10.1016/j. ejmp.2007.07.001, indexed in Pubmed: 17825595.

13. Sanchez-Nieto B, Nahum AE. BIOPLAN: software for the biological evaluation of. Radiotherapy treatment plans. Med Dosim. 2000; 25(2): 71-76, doi: 10.1016/s09583947(00)00031-5, indexed in Pubmed: 10856684.

14. Zhou ZR, Mei X, Chen XX, et al. Systematic review and meta-analysis comparing hypofractionated with conventional fraction radiotherapy in treatment of early breast cancer. Surg Oncol. 2015; 24(3): 200-211, doi: 10.1016/j. suronc.2015.06.005, indexed in Pubmed: 26116397.

15. Dearnaley D, Syndikus I, Mossop H, et al. CHHiP Investigators. Conventional versus hypofractionated high-dose intensity-modulated radiotherapy for prostate cancer: 5-year outcomes of the randomised, non-inferiority, phase 3 CHHiP trial. Lancet Oncol. 2016; 17(8): 1047- 
1060, doi: 10.1016/S1470-2045(16)30102-4, indexed in Pubmed: 27339115.

16. Budach W, Bölke E, Matuschek C. Hypofractionated Radiotherapy as Adjuvant Treatment in Early Breast Cancer. A Review and Meta-Analysis of Randomized Controlled Trials. Breast Care (Basel). 2015; 10(4): 240-245, doi: 10.1159/000439007, indexed in Pubmed: 26600759.

17. Miller $A B$, Wall C, Baines $C J$, et al. Twenty five year follow-up for breast cancer incidence and mortality of the Canadian National Breast Screening Study: randomised screening trial. BMJ. 2014;348: g366, doi: 10.1136/bmj.g366, indexed in Pubmed: 24519768.

18. Barnett GC, Coles CE, Elliott RM, et al. Independent validation of genes and polymorphisms reported to be associated with radiation toxicity: a prospective analysis study. Lancet Oncol. 2012; 13(1): 65-77, doi: 10.1016/ S1470-2045(11)70302-3, indexed in Pubmed: 22169268.

19. AkI F, Khater A. Hypofractionated versus Conventionally Fractionated Radiotherapy in Post-Mastectomy Breast Cancer Patients. J Cancer Ther. 2018; 09(11): 941-954, doi: 10.4236/jct.2018.911078.

20. De Felice F, Ranalli T, Musio D, et al. Relation between Hypofractionated Radiotherapy, Toxicity and Outcome in Early Breast Cancer. Breast J. 2017; 23(5): 563-568, doi: 10.1111/tbj.12792, indexed in Pubmed: 28252236.
21. Gloi A. A Broad Evaluation of Left Breast Radiotherapy. Am J Biomed Sci. 2019: 152-171, doi: 10.5099/aj190200153.

22. Hegemann NS, Guckenberger M, Belka C, et al. Hypofractionated radiotherapy for prostate cancer. Radiat Oncol. 2014; 9: 275, doi: 10.1186/s13014-014-0275-6, indexed in Pubmed: 25480014.

23. Mazonakis M, Stratakis J, Lyraraki E, et al. Risk of contralateral breast and ipsilateral lung cancer induction from forward-planned IMRT for breast carcinoma. Phys Med. 2019;60:44-49, doi: 10.1016/j.ejmp.2019.03.021, indexed in Pubmed: 31000085.

24. Gokula K, Earnest A, Wong LC. Meta-analysis of incidence of early lung toxicity in 3-dimensional conformal irradiation of breast carcinomas. Radiat Oncol. 2013; 8: 268, doi: 10.1186/1748-717X-8-268, indexed in Pubmed: 24229418.

25. Duane FK, McGale P, Brønnum D, et al. Risk of ischemic heart disease in women after radiotherapy for breast cancer. N Engl J Med. 2013; 368(11): 987-998, doi: 10.1056/ NEJMoa1209825, indexed in Pubmed: 23484825.

26. Graham MV, Purdy JA, Emami B, et al. Clinical dose-volume histogram analysis for pneumonitis after 3D treatment for non-small cell lung cancer (NSCLC). Int J Radiat Oncol Biol Phys. 1999; 45(2): 323-329, doi: 10.1016/s03603016(99)00183-2, indexed in Pubmed: 10487552. 